\title{
INTEGRATION IN ABSTRACT SPACES
}

\section{T. H. HILDEBRANDT}

In thinking about possible subjects for this address, I could not help thinking back to the first "invited address" which I gave before members of the Society. The address together with one given by the late G. A. Bliss was the basis of the first Symposium of what was then called the Chicago Section of the Society, the subject Lebesgue integration, the time April 1917. These symposia were initiated because of the desire of the Chicago Section to make their meetings more interesting. It was felt that if an occasional session could be devoted to a single topic, with one or two speakers, and an outline of and information concerning the material could be sent out in connection with the program of the meeting, it would make effective audience participation possible and prove more profitable and stimulating to members of the Society. The idea was taken up by other groups and now takes the form of an "invited address," where unfortunately the audience does not have advance preparation, and stimulating discussion is avoided. I note that in recent years we have had committees to consider ways and means of making the sessions of the Society more effective, and we shall probably have this problem always with us. I do feel that the system of invited addresses has proven itself in that it has been worth while for him who gives and him who takes, particularly when the address results in published summaries of research covering a vital field.

For me that first symposium was very much worth while; it aroused and established in me an interest in integration. So a first suggestion for this address was to give a summary of what has happened in integration since that time. But the immensity of such a project is obvious, and the impossibility of including such within the scope of an address no less so. I have, consequently, narrowed my field, and expect to speak on two rather narrow lines of generalization of integration, with emphasis on Lebesgue integration, one in the direction of linear spaces, the other using the order character of some linear spaces.

1. The Lebesgue integration postulates. It might be interesting to recall briefly the axiomatic program which Lebesgue set up, and which led him to the now well known definition of measurable sets

Retiring presidential address delivered at the Athens, Georgia, meeting of the Society on December 31, 1947; received by the editors June 26, 1952. 
and integration as given in his book Sur l'integration (first edition (1904) pp. $98 \mathrm{ff} .$, 2nd edition, pp. $105 \mathrm{ff}$.). He proposes to define an integration process yielding a real number for any of the class $\mathfrak{F}$ of bounded real valued functions $f$, and any finite interval $a \leqq s \leqq b$, subject to the following conditions.

(1) For all $a, b$, and $h: \int_{a}^{b} f(s) d s=\int_{a+h}^{b+h} f(s-h) d s$.

(2) For all $a, b, c: \int_{a}^{b} f+\int_{b}^{c} f+\int_{a}^{a} f=0$.

(3) For all $f_{1}$ and $f_{2}: \int_{a}^{b}\left(f_{1}+f_{2}\right)=\int_{a}^{b} f_{1}+\int_{a}^{b} f_{2}$.

(4) If $f \geqq 0$ and $b>a$, then $\int_{a}^{b} f \geqq 0$.

(5) $\int_{0}^{1} 1 d s=1$.

(6) If $f_{n}(s) \leqq f_{n+1}(s)$ for all $n$ and $s$ and $\lim _{n} f_{n}(s)=f(s)$ for all $s$, then $\lim _{n} \int_{a}^{b} f_{n}=\int_{a}^{b} f$.

His analysis of these properties yields among other things that if $b>a$, then (3) and (4) imply (a) $\left|\int_{a}^{b} f\right| \leqq \int_{a}^{b}|f|$; (b) $\int_{a}^{b} c f=c \int_{a}^{b} f$ for all real $c$, so that the integral is linear on the class $\mathfrak{F}$; (c) if $f_{n}$ approaches $f$ uniformly on $(a, b)$, then $\lim _{n} \int_{a}^{b} f_{n}=\int_{a}^{b} f$. Consequently, the properties (3) and (4) require that $\int_{a}^{b} f$ with $b>a$ be a positive linear continuous functional or form on the space $\mathfrak{F}$ of all bounded functions, the least upper bound of the absolute value being the norm of the function. By dividing up the range of values of $f(s)$, Lebesgue notes that his problem would be solved if he knew the value of the integral for the characteristic functions of all subsets of the linear interval. It is remarkable how closely Lebesgue approached the solution of the problem of obtaining the most general linear continuous functional or form of the space of bounded functions on a finite or infinite interval. A solution of this latter problem was published much later, first by the speaker [30] and shortly thereafter by Fichtenholz and Kantorovic [18], viz., that such a form is completely determined by a bounded additive function of all subsets of the interval. It can be expressed in the form $\int f(s) d \alpha(E)$ where the integration is definable either by the Lebesgue process of subdividing the range of $f(s)$ or by successive subdivisions or partitions of the interval into a finite number of disjoint sets. The positive property (4) requires that $\alpha(E)$ $\geqq 0$ for all $E$, i.e., $\alpha(E)$ is a positive finitely additive measure function on all subsets of the interval. Turning to the other conditions imposed by Lebesgue, the additional continuity restriction involved in condition (6) when applied to $\int f d \alpha$ requires that if $E_{n}$ is a monotonic nonincreasing sequence of sets without common element, then $\lim _{n} \alpha\left(E_{n}\right)=0$, which in turn is equivalent to the complete additivity of the measure function $\alpha(E)$ on all sets. Now condition (1) applied to a measure function requires that it be invariant under translation, 
and we have here the precursor of the Haar-measure problem, to determine a completely additive measure function on a completely additive class of sets, invariant under translation. Conditions (2) and (3) demand that, for intervals, this measure should reduce to the length of the interval. At this point Lebesgue abandons the project of determining an integral satisfying conditions (1) to (6) for all bounded functions, and sets up a process for determining what is now well known as the class of measurable subsets of the straight line, a measure function on these sets, and a class of measurable functions. Banach ([2] and [3, pp. 30-32]) has given a procedure for finding an integral for all bounded functions on $0 \leqq s \leqq 1$, satisfying conditions (1) to (5) but not (6). This would correspond to a bounded finitely additive invariant measure on all subsets of $0 \leqq s \leqq 1$. So far as I am aware, no one has settled the question whether the set of Lebesgue measurable bounded functions is the largest subclass of these functions, with an integral satisfying all of Lebesgue's postulates.

One might consider a corresponding set of postulates for Stieltjes integrals and their generalization in which the emphasis would be on the bilinear character of these integrals. Thus one might ask for a number corresponding to every pair of bounded functions $f$ and $g$ and interval $a \leqq s \leqq b$, denoted by $S(f, g ; a, b)$ reducing to say the Riemann-Stieltjes integral when the latter exists. There would be no postulate corresponding to (1). Condition (2) would go over unchanged. Condition (3) would require bi-additivity in $f$ and $g$. Condition (4) would take the form: if $f \geqq 0, g$ is monotonic non-decreasing, and $b>a$, then $S(f, g ; a, b) \geqq 0$. Condition (5) would be $S(1, g ; a, b)$ $=g(b)-g(a)$. Condition (6) might involve two convergence properties: (a) if $f_{n}(s) \leqq f_{n+1}(s)$ for all $n$ and $s$, and $\lim _{n} f_{n}(s)=f(s)$ for all $s$, then for every monotone function $g, \lim _{n} S\left(f_{n}, g ; a, b\right)=S(f, g ; a, b)$; (b) if $g_{n}(s)$ are monotone nondecreasing, and $\lim _{n} g_{n}(s)=g(s)$ for all $s$, then $\lim _{n} S\left(f, g_{n} ; a, b\right)=S(f, g ; a, b)$.

We might recall in passing that Lebesgue's definition of an integral is essentially a Stieltjes integral. If $f$ is measurable and we set $\mu(y)$ $=$ meas $E(s \mid f(s)<y)$, then $L \int_{a}^{b} f(s) d s=S \int_{-\infty}^{\infty} y d \mu(y)$, convergence of the right-hand side being necessary and sufficient for the integrability of $f$, when $f$ is not bounded. This form emphasizes the distributional character of integration. As is well known, there are other approaches to Lebesgue integration, some of which will be mentioned later, the amazing, or perhaps gratifying, fact is that so many different approaches lead to the same thing in the end, justifying Lebesgue's solution. 
2. The Fréchet general integral. The extension of Lebesgue integration in which the functions are defined on an $n$-dimensional rectangle instead of a linear interval was an easy one. The Radon suggestion of replacing $n$-dimensional measure by a positive completely additive set function was the basis of the natural generalization made by Fréchet [20]. In this generalization the linear interval is replaced by a general set $\subseteq$, measurable subsets are postulated to be a $\sigma$-field (5 of subsets $E$ (satisfying the conditions (a) if $E_{n}, n=1,2, \cdots$, belong to $\left(\xi\right.$, so does the set sum or join $\sum_{n} E_{n}$; (b) if $E_{1}$ and $E_{2}$ belong to $\left(\mathbb{E}\right.$, so does $E_{1}-E_{2}$; (c) $\subseteq$ belongs to $(\mathbb{E})$. Measure is replaced by a set function, $\alpha(E)$, on $\&$ to real numbers, completely additive on $\left(\xi\right.$, i.e., if $E=\sum_{n} E_{n}$ in $\$$, and $E_{n}$ are disjoint, then $\alpha\left(\sum_{n} E_{n}\right)=\sum_{n} \alpha\left(E_{n}\right)$. Then $\alpha$ is bounded on $\&$, has a total variation $\int|d \alpha|$ on all subsets of $\xi$, and can be written as the difference of two positive-valued completely additive set functions. A function is measurable relative to $\&$ if the set $E(s \mid f(s)<a)$ belongs to $₹$ for all real numbers $a$. Then the Lebesgue procedure can be applied relative to each of the positive parts of $\alpha$, yielding $\int f d \alpha$. Fréchet also called attention to the applicability of a method of defining an integral following the ideas suggested by W. H. Young, by introducing upper and lower integrals. For this it is necessary to assume that there exists a subdivision of $\mathbb{S}$ into a denumerably infinite number of sets of $\left(\xi\right.$, so that $\sum_{n} M_{n} \tau\left(E_{n}\right)$ converges, where $\tau(E)$ is the total variation of $\alpha$ on $E$, and $M_{n}$ is the least upper bound of $|f|$ on $E_{n}$. Then finite-valued upper and lower integrals based on subdivisions of $\sqrt{ }$ into sets of $\sqrt{ }$ exist, equality giving rise to an integral. An alternative procedure is possible by utilizing the Moore-Smith type of limit [45]. For this, subdivisions $\pi$ of $\subseteq$ are ordered by inclusion, $\pi_{1} \geqq \pi_{2}$ meaning that every subset of $\pi_{1}$ is part of a subset of $\pi_{2}$. Then $f(s)$ has an integral with respect to $\alpha(E)$ if $\lim _{\pi} \sum_{n} f\left(s_{n}\right) \alpha\left(E_{n}\right)$ exists, $s_{n}$ being in $E_{n}$. Since a subdivision does not define any order in the sets $E_{n}$ composing it, the infinite series involved must converge absolutely. The methods of Lebesgue and Young have the same set of integrable functions if the class of measurable sets is complete relative to $\alpha$, i.e., if $\int_{E}|d \alpha|=0$, and $E_{0}$ is contained in $E$, then $E_{0}$ is in $\leftleftarrows$ and $\int_{E_{0}}|d \alpha|=0$.

\section{INTEGRALS IN LINEAR NORMED SPACES}

3. Riemann integrals. The next most obvious generalization of the integration process consists in extending the value space of the functions to be integrated and the integral values to the simplest generalizations of the space of real numbers, viz., a linear normed com- 
plete or LNC or Banach space. ${ }^{1}$ A space $\mathfrak{X}$ of elements $x$ is linear if addition as a commutative group and multiplication by real (or complex) numbers is defined. A linear space is normed if every $x$ has a norm $\|x\|$ satisfying the conditions: $\|x\| \geqq 0$ for all $x ;\|x\|=0$ is equivalent to $x=0$ (the null element of the space); and $\left\|a_{1} x_{1}+a_{2} x_{2}\right\|$ $\leqq\left|a_{1}\right|\left\|x_{1}\right\|+\left|a_{2}\right|\left\|x_{2}\right\|$ for all numbers $a_{1}, a_{2}$ and $x_{1}, x_{2}$ of $\mathfrak{X}$. A normed space is complete if for a sequence $x_{n}$ such that $\lim _{m, n}\left\|x_{m}-x_{n}\right\|=0$ there exists an $x$ such that $\lim _{n}\left\|x_{n}-x\right\|=0$.

The simplest integration notion in such an LNC space $\mathfrak{X}$ is that of the Riemann integral of a function $x(s)$ on the linear interval $a \leqq s \leqq b$ to $\mathfrak{X}$, as the limit in the space, of sum $\sum_{i=0}^{n-1} x\left(s_{i}^{\prime}\right)\left(s_{i+1}-s_{i}\right)$, where $a=s_{0}<s_{1}<\cdots<s_{n}=b$ is a subdivision of $(a, b), s_{i} \leqq s_{i}^{\prime} \leqq s_{i+1}$, and the limit is taken as the norm of the subdivision, the maximum of $s_{i+1}-s_{i}$ approaches zero. This generalization is due to L. M. Graves [27]. The proof of the integrability of a function $x(s)$ continuous on $(a, b)$ follows the usual pattern, but the boundedness of the function $\|x(s)\|$ and its Riemann integrability, while sufficient, are not necessary for the integrability of a function $x(s)$. Simple counter examples are available in the space of bounded sequences.

The extension of these notions to Riemann Stieltjes integrals is conceptually simple. There are two possibilities: (a) the integration of a function $x(s)$ on $a \leqq s \leqq b$ to $\mathfrak{X}$ with respect to a real-valued function $g(s)$; (b) the integration of a real-valued function $f(s)$ with respect to a function $x(s)$ to $\mathfrak{X}$ (for the latter see VanderLijn, G. [62]). The limit of the approximating sums $\sum_{i=0}^{n-1} x\left(s_{i}^{\prime}\right)\left(g\left(s_{i+1}\right)-g\left(s_{i}\right)\right)$ or $\sum_{i=0}^{n-1} f\left(s_{i}^{\prime}\right)\left(x\left(s_{i+1}\right)-x\left(s_{i}\right)\right)$ is possible either as the $|\sigma|$ $=\max _{i}\left(s_{i+1}-s_{i}\right)$ approaches zero, or by successive subdivisions, $\sigma_{1} \geqq \sigma_{2}$ if $\sigma_{1}$ contains all of the points of $\sigma_{2}$. Obviously an integration by parts theorem between the two types of integrals holds, i.e., $\int_{a}^{b} x(s) d g(s)$ and the corresponding $\int_{a}^{b} g(s) d x(s)$ exist simultaneously and their sum is $x(b) g(b)-x(a) g(a)$. The integral (a) exists if $x(s)$ is continuous and $g(s)$ is of bounded variation, and the integral (b) exists if $f(s)$ is continuous and $x(s)$ is strongly of bounded variation in the sense that $\sum_{i=0}^{n-1}\left\|x\left(s_{i+1}\right)-x\left(s_{i}\right)\right\|$ is bounded as a function of the subdivision $\sigma$ of $(a, b)$. Dunford [17, p. 312] has shown that if $f(s)$ is continuous and $x(s)$ is weakly of bounded variation in the sense

${ }^{1}$ We shall use the terminology linear normed complete or LNC space in preference to Banach space for two reasons: (a) the concept of such spaces predated Banach's formulation (see F. Riesz, Acta Math. vol. 41 (1916) pp. 72, 73; A. A. Bennett, Proc. Nat. Acad. Sci. U.S.A. vol. 21 (1916) p. 595; K. W. Lamson, Amer. J. Math. vol. 42 (1920) p. 245), (b) there are linear spaces in which the topology is different, and it seems better to include in the name of the space its mathematical character. 
that if $\sigma$ consists of the finite disjoint subintervals $\left(s_{1}^{\prime}, s_{1}^{\prime \prime}\right), \cdots$, $\left(s_{n}^{\prime}, s_{n}^{\prime \prime}\right)$, then $\| \sum_{i=1}^{n}\left(x\left(s_{i}^{\prime}\right)-x\left(s_{i}^{\prime \prime}\right) \|\right.$ is bounded in $\sigma$, then $\int_{a}^{b} f(s) d x(s)$ exists, and this condition is necessary if we want the integral to exist for all continuous functions. Stieltjes integrals of type (a) occur in the resolution of the identity of self-adjoint transformations in Hilbert spaces.

4. The Bochner integral. The definition of measurability and integrability for $x(s)$ on an $n$-dimensional interval $\mathfrak{S}$, to an LNC space due to Bochner [6], has proved very effective. A function $x(s)$ is measurable if it is the limit almost everywhere of a sequence of finite-valued measurable functions, a function $x(s)$ being a finitevalued measurable function if there exists a subdivision of $\subseteq$ into a finite number of disjoint measurable subsets, $E_{1}, \cdots, E_{n}$, on each of which $x(s)$ is constant. If $\mathfrak{X}$ is the real number system, the definition of measurability yields the usual set of measurable functions. The function $x(s)$ is integrable on $\subseteq$ if $x(s)$ is measurable and $\|x(s)\|$, which is therefore also measurable, has a finite Lebesgue integral on $\mathfrak{S}$. It is shown that it is then possible to assign a unique value to $\int x(s) d s$ as the limit of $\int x_{n}(s) d s$, where the $x_{n}(s)$ are finite-valued functions approaching $x(s)$ almost everywhere with $\left\|x_{n}(s)\right\| \leqq f(s)$, $f(s)$ some integrable function on $\mathfrak{S}$, and $\int x_{n}(s) d s=\sum_{i=1}^{k} x_{n i} m\left(E_{n i}\right)$, $x_{n}(s)=x_{n i}$ on $E_{n i}$. The limit of the sequence of integrals is independent of the sequence of finite-valued functions satisfying the given conditions. The resulting integral has the usual properties of Lebesgue integration; for instance, the complete additivity of $\int_{E} x(s)$ as a function of measurable sets, and the convergence property: if the $x_{n}(s)$ are integrable, converge to $x(s)$ almost everywhere, and $\left\|x_{n}(s)\right\| \leqq f(s)$, for all $n$ and $s$, with $f(s)$ integrable, then $\lim _{n} \int_{E} x_{n}(s) d s=\int_{E} x(s) d s$ for all measurable sets $E$ of $\mathfrak{S}$.

5. The Dunford (first) integral. A clever new point of view was injected into the theory of Lebesgue integration by Dunford [15] who observed that if one normed the space of continuous functions by the condition $\|f\|=\int|f(s)| d s$, the resulting linear normed space is not complete. If one completes this metric space in the usual way, one gets exactly the Lebesgue integrable functions. By changing the norm of $f$ to $\|f\|=\left.\left.\left|\int\right| f(s)\right|^{p} d s\right|^{1 / p}, p \geqq 1$, the space $L^{p}$ of functions for which $|f(s)|^{p}$ is integrable emerges. Adapting this technique, Dunford assumes a completely additive set function $\alpha$ defined on a $\sigma$-field of subsets of $n$-dimensional Euclidean space, which includes the Borel measurable sets. It is shown that if $x(s)$ is continuous on $\mathfrak{S}$, then $\int x(s) d \alpha(E)$ as well as $\int\|x(s)\| d \beta(E)$ exist, where $\beta(E)$ is the 
total variation of $\alpha(E)$ on $E$. Then the class of Lebesgue integrable functions $x(s)$ is the completion of the class of continuous functions on $\subseteq$ normed by $\int\|x(s)\| d \beta(E), x(s)$ is the limit of a sequence of continuous functions almost everywhere in terms of $\beta$, and $\int x(s) d \alpha(E)$ is the limit of $\int x_{n}(s) d \alpha(E)$, where $x_{n}(s)$ is any sequence of continuous functions such that $\lim _{n} \int\left\|x_{n}(s)-x(s)\right\| d \beta(E)=0$. In the case in which $\alpha(E)$ is Lebesgue measure, the class of integrable functions as well as their integrals agree with that of the Bochner definition. On the other hand, there are Riemann integrable functions for which $\int\|x(s)\| d s=\infty$, which are therefore not integrable by either of these definitions.

Dunford extends this definition to a metric space, probably because he started with an integral on the space of continuous functions. Later he noticed that a more elegant approach is via the space of finite-valued measurable functions, since this does not involve any topological conditions on $\widetilde{S}$. The next step is then obvious: we take a general space $\mathfrak{S}$, a class of (measurable subsets) $\mathbb{E}$, which forms a $\sigma$-field, a completely additive set function $\alpha$ on $\&$ to real numbers, and define an integral for the class of finite-valued measurable functions. We norm this class of functions with $\int\|x(s)\| d \beta(E)$, where $\beta(E)$ is the total variation of $\alpha$ on $E$. The completion of this class of functions under this norm gives the Lebesgue integrable functions $L(\mathfrak{X})$ as well as an integral.

6. Other approaches to Bochner integral. As we have already noted, the building up of an integral of a function $x(s)$ on $\subseteq: a \leqq s \leqq b$ to an LNC space $\mathfrak{X}$, following the Lebesgue process runs into the difficulty that his definition of measurable functions is dependent on an order process, while no such order is postulated in $\mathfrak{X}$. Hence, other approaches are necessary. The speaker, in an unpublished paper [31] predating Bochner and Dunford, noted that the process outlined by F. Riesz [58] for the definition of a Lebesgue integral could be carried over to the more general setting without much change. Defining a step function on $(a, b)$ in the usual way, i.e., $x(s)$ $=x_{i}$ for $s_{i} \leqq s<s_{i+1} ; x(b)=x_{n}$, for the subdivision $\sigma=\left(a=s_{0}<s_{1}<\ldots\right.$ $\left.<s_{n}=b\right)$ of $(a, b)$ and $\int x(s) d s=\sum_{i=1}^{n} x_{i}\left(s_{i+1}-s_{i}\right)$, an arbitrary bounded function $x(s)$ on $(a, b)$ is integrable if it is the limit of a sequence of step functions almost everywhere, the $\int x(s) d s$ being the limit of $\int x_{n}(s) d s$ where $x_{n}(s)$ is any bounded sequence of step functions converging to $x(s)$ almost everywhere. In the case when $x(s)$ is unbounded, the sequence of step functions $x_{n}(s)$ converging to $x(s)$ is subject to the additional condition that $\int_{E} x_{n}(s) d s$ be uniformly ab- 
solutely continuous in the sense that $\int_{E}\left\|x_{n}(s)\right\| d s$ are uniformly absolutely continuous. It follows then that $\int_{a}^{b}\|x(s)\| d s$ exists, so that this procedure is equivalent to that of Bochner.

An alternative approach is suggested by the definition of Lebesgue integral due to Hahn [28]. A function $x(s)$ is defined to be measurable if for every $\epsilon>0$, there exists a perfect set $P_{\epsilon}$, such that the measure of $P_{\epsilon}$ is greater than $b-a-\epsilon$, and $x(s)$ is continuous on $P_{\epsilon}$. This uses the Lusin property of measurable functions. A Riemann type of integral is obviously definable on $P_{\epsilon}$. If $x(s)$ is bounded and measurable in this sense on $(a, b)$, then $\int x(s) d s=\lim _{\epsilon \rightarrow 0} \int_{P_{\epsilon}} x(s) d s$, the $P_{\epsilon}$ being limited to the perfect sets on which $x(s)$ is continuous. This limit can be shown to exist. If $x(s)$ is unbounded, then it is integrable on $(a, b)$ if $\lim _{\epsilon \rightarrow 0} \int_{P_{\epsilon}} x(s) d s$ exists, with the same limitation on $P_{\epsilon}$. While under these hypotheses $\|x(s)\|$ is a real-valued measurable function, it is sufficient but not necessary that it be integrable in the Lebesgue sense, in order to have $x(s)$ integrable according to this definition.

7. Birkhoff integral. For some reason or other the Young approach used by Fréchet in setting up an integral for functions on a general space was passed over. In order to make effective use of this procedure, it was necessary to extend the notion of unconditional convergence of an infinite series of elements to an LNC space $\mathfrak{X}$. Birkhoff [4] defines this as convergence to the same element by any rearrangement of the series. There are several equivalent methods of defining unconditional convergence (see, e.g., this Bulletin vol. 46 (1940) p. 950), the most elegant method being based on a Moore-Smith limit. If $\pi$ represents a finite subset: $\left(n_{1}, \cdots, n_{k}\right)$ of the integers and $\pi_{1} \geqq \pi_{2}$ is defined by set inclusion, then the $\pi$ form a directed set. $\sum x_{n}$ is unconditionally convergent if $\lim _{\pi} \sum_{\pi} x_{n}$ exists. If $\mathfrak{X}$ is the space of real or complex numbers, then an unconditionally convergent series is absolutely convergent. It is obviously possible to define in a similar way the meaning of $\sum_{q} x_{q}$, where the $q$ are any general set $\mathfrak{Q}$ (no order needed), by setting $\pi=\left(q_{1}, \cdots, q_{k}\right)$ and defining $\sum_{q} x_{q}$ as the limit as $\pi$ spreads of $\sum_{\pi} x_{q}$. It is easily demonstrable that $\sum_{q} x_{q}$ exists if and only if $x_{q}$ vanishes except for a denumerably infinite subset of $\mathfrak{Q}$, and $\sum_{n} x_{q_{n}}$ extended over this subset is unconditionally convergent. Birkhoff $[4$, p. 362$]$ notes that the set of unconditionally convergent sequences of an LNC space $\mathfrak{X}$ form again an LNC space. The notion of unconditional convergence is applicable to any sequence of sets. If $X_{1}, \cdots, X_{n}, \cdots$ are subsets of $\mathfrak{X}$, then $\sum X_{n}$ is unconditionally convergent if $\sum x_{n}$ is unconditionally convergent 
for any choice of the $x_{n}$ in $X_{n}$. The class of elements so expressible is defined as $\sum_{n} X_{n}$.

For the definition of an integral, Birkhoff [4] assumes that the general set $\subseteq$ contains a $\sigma$-field $(E$ of "measurable" subsets $E, \alpha(E)$ is a positive completely additive function on $(\xi$, and $x(s)$ is a point function on $\mathfrak{S}$ to $\mathfrak{X}$. Then subdivisions $\sigma$ of $\mathfrak{S}$ into disjoint sets $E_{1}, \cdots, E_{n}, \cdots$ of determines the set of elements $X(x, \sigma)$ $=\sum_{n} x\left(s_{n}\right) \alpha\left(E_{n}\right)$, with $s_{n}$ in $E_{n}$, if the series of sets is unconditionally convergent. The closed convex extension of $X(x, \sigma)$ denoted by $\mathrm{Cl}$ co $X(x, \sigma)$ is called an integral range of $x(s)$ corresponding to $\sigma$. This is a generalization of the interval defined by the extremes of approximating sums in the case of real numbers. If the greatest lower bound of the diameter of $\mathrm{Cl} \operatorname{co} X(x, \sigma)$ relative to $\sigma$ is zero, $x(s)$ is said to be integrable, the integral being the common point of $\mathrm{Cl}$ co $X(x, \sigma)$. By the use of the Moore-Smith limit it is possible to by-pass the convex extension and closure. If one orders subdivisions $\sigma$ of $\subseteq$ by assuming that $\sigma_{1} \geqq \sigma_{2}$ if $\sigma_{1}$ is finer than $\sigma_{2}$ (every subset of $\sigma_{1}$ is a subset of some set of $\left.\sigma_{2}\right)$ then $x(s)$ is integrable with respect to the completely additive set function $\alpha(E)$ if the $\lim _{\sigma} \sum_{n} x\left(s_{n}\right) \alpha\left(E_{n}\right)$ exists. A necessary condition for the existence of such an integral is that there exist a subdivision $\sigma_{0}$ such that for any $\sigma \geqq \sigma_{0}, \sum_{\sigma} x\left(s_{n}\right) \alpha\left(E_{n}\right)$ be unconditionally convergent for any choice of the $s_{n}$ in $E_{n}$. If the Birkhoff integral exists on $\mathfrak{S}$, it also exists on every subset $E$ of $\mathbb{E}$ and is a completely additive set function on $\mathbb{E}$ to $\mathfrak{X}$, in the sense that if $E=\sum E_{n}\left(E_{n}\right.$ disjoint $)$ then $x(E)=\sum_{n} x\left(E_{n}\right)$, the series on the right being unconditionally convergent. The Birkhoff integral includes the Riemann type of integral, as well as the Bochner and Dunford integrals as special cases, and gives an integral value for some functions which are not integrable by these other methods.

In reading the Birkhoff paper, one notices that he could also have defined an alternative type of integral, where $f(s)$ is a real-valued point function and $x(E)$ is a completely additive set function on to $\mathfrak{X}$ as defined in the preceding paragraph. Most of the reasoning applies to yield integrals of the form $\int f(s) d x(E)$. It has bilinear properties in $f$ and $x(E)$.

8. Bilinear integrals. Once the observation has been made that either the point function or the set function may belong to an LNC space $\mathfrak{X}$ and one recalls that the space of real numbers is an LNC space, the idea of having both of these functions in LNC spaces is imminent. But now the question of multiplication arises. We either enter the field of ring spaces, or observe that the product of a real 
number into an element $x$ of $\mathfrak{X}$ is a bilinear transformation on the product space $\mathfrak{A X}$ to $\mathfrak{X}$ ( $\mathfrak{A}$ being the space of real numbers). We propose to replace multiplication by real numbers by a bilinear transformation $B$ on the product space $\mathfrak{X} \mathfrak{Y}$ to $\mathfrak{Z} ; \mathfrak{X}, \mathfrak{Y}$, and $\mathfrak{Z}$ being LNC spaces. It is conceivable that one may need a boundedness condition, i.e., that there exists an $M \geqq 0$, such that $\|B(x, y)\| \leqq M\|x\|\|y\|$. Assuming, then, such a bilinear transformation, a point function $x(s)$ on $\subseteq$ to $\mathfrak{Y}$, a set function $y(E)$ on the sets $E$ of $\mathbb{E}$ to $\mathfrak{X}$, we can follow the usual pattern and define $\int B(x(s), d y(E))$ as that element of 3 which is equal to $\lim _{\sigma} \sum_{\sigma} B\left(x\left(s_{n}\right) y\left(E_{n}\right)\right)$ provided the limit exists, the series involved being assumed to be unconditionally convergent. Bilinearity of $B$ suffices to make this integral bilinear in $x(s)$ and $y(E)$. Boundedness of $B(x, y)$ in $y$ for each $x$ and complete additivity of the set function $y(E)$ are sufficient to guarantee that if $x$ is a finite-valued measurable function then $\int B(x(s), d y(E))$ exists and is equal to $\sum_{i} B\left(x_{i}, y\left(E_{i}\right)\right), x_{i}$ being the value of $x(s)$ on $E_{i}$. Other conditions are needed to guarantee complete additivity of the integral as a set function or the interchangeability of integral and limits.

Instances of this type were considered by Gowurin [26] and by Bochner and Taylor [8]. Gowurin assumes that $\subseteq$ is the linear interval $(a, b)$ and $\mathfrak{V}$ the space of linear bounded transformations $T$ on the LNC space $\mathfrak{X}$ to the LNC space $\mathfrak{Z}$. Then $\mathfrak{Y}$ is a linear normed complete space under the modulus of $T, B(y, x)=T x$ is a bounded bilinear transformation on $\mathfrak{Y X}$ to $\mathbb{Z}$ with $\|B(y, x)\| \leqq\|T\|\|x\|$. Conversely if $B(y, x)$ is a bounded bilinear transformation on $\mathfrak{X Y Y}$ to $\mathbb{Z}$, then for fixed $y, B(y, x)$ defines a linear bounded transformation on $\mathfrak{X}$ to $\mathbb{Z}$, i.e. $B(y, x)$ as $y$ ranges over $\mathfrak{Z}$ defines a subset of the linear bounded transformations on $\mathfrak{X}$ to $\mathfrak{Z}$. If $y(s)$ and $x(s)$ are point functions on $\subseteq$ to $\mathfrak{Y}$ and $\mathfrak{X}$ respectively, we can set up the Stieltjes sums $\sum_{\sigma} B\left[y\left(s_{i}^{\prime}\right) ; x\left(s_{i+1}\right)-x\left(s_{i}\right)\right]$, where $\sigma=\left(a=s_{0} \leqq s_{1} \leqq s_{2} \leqq \cdots \leqq s_{n}=b\right)$ is a subdivision of $(a, b)$ and $s_{i} \leqq s_{i}^{\prime} \leqq s_{i+1}$. If these sums have a limit either as the $\max \left(s_{i+1}-s_{i}\right)$ approaches zero or as $\sigma$ spreads, we get a Stieltjes integral $\int_{a}^{b} B[y(s) ; d x(s)]$. This Stieltjes integral possesses bilinear properties in $x$ and $y$ as well as an integration by parts theorem. In order to obtain the existence of this integral for all continuous functions $y(s)$ on $\subseteq$ to $\mathfrak{Y}$, Gowurin proposes a generalization of the notion of bounded variation. The function $x(s)$ on $\subseteq$ to $\mathfrak{X}$ has finite $W$-variation relative to $B$ and $\mathfrak{Y}$, if there exists a positive constant $M$ such that $\left\|\sum_{\sigma} B\left[y_{i} ; x\left(s_{i+1}\right)-x\left(s_{i}\right)\right]\right\| \leqq M \max _{i}\left\|y_{i}\right\|$ for all subdivisions $\sigma$ of $(a, b)$ and all $y_{1}, \cdots, y_{n}$ of $\mathfrak{Y}$. This condition on $x(s)$ is necessary and sufficient to guarantee the existence of $\int B[y(s) ; d x(s)]$ for all continuous functions $y(s)$ if $B$ is bilinear bounded. It may be 
noted that if $\mathfrak{Y}=\mathfrak{X}^{*}$, the space of linear continuous forms on $\mathfrak{X}$, and $B$ is the set of real numbers, then $x(s)$ is of finite $W$-variation if and only if it is of strong bounded variation on $\mathfrak{S}$; if $\mathfrak{V}=\mathfrak{A}$, the space of reals, and $\mathbb{B}=\mathfrak{X}$, then finite $W$-variation reduces to weak bounded variation. These considerations find their application in the problem of finding the form of the most general linear forms on what might be called second story spaces, whose elements are functions on a range to an LNC space.

9. The Price integral. The Gowurin integrals are essentially of the Riemann type. Price [54] has made an extensive study of the corresponding situation using instead the Young-Birkhoff slant. The real-valued measure function $\mu(E)$ is replaced by a function on the class of "measurable" sets to the space $\mathfrak{I}$ of linear bounded transformations $\tau$ on the LNC space $\mathfrak{X}$ to $\mathfrak{X}$ with $\|\tau\|=\operatorname{LUB}[\|\tau(x)\|$ for $\|x\|=1]$. The following properties of $\tau(E)$ generalize the measure function properties: (a) if $\tau\left(E_{0}\right)=0$, and $E \leqq E_{0}$, then $\tau(E)=0$; (b) if $\tau(E) \neq 0$ then $\tau(E)$ has a reciprocal in $\mathfrak{T}$; (c) $\tau(E)$ is completely additive on $\&$, i.e., if $\sum_{n} E_{n}=E$ in (E, $E_{n}$ disjoint, then $\tau\left(\sum_{n} E_{n}\right)$ $=\sum_{n} \tau\left(E_{n}\right)$, with unconditional convergence. One could obviously follow the Birkhoff path and consider sums $\sum \tau\left(E_{n}\right) x\left(s_{n}\right)$ with $s_{n}$ in $E_{n}$ over subdivisions into measurable subsets of $\mathfrak{S}$. Price prefers to inject a generalization of the notion of the convex extension of a set which plays a strong role in Birkhoff's definition. The generalization rests on the observation that the convex extension of a set $X_{0}$ is the totality of elements of the form $\sum_{1}^{n} c_{i} x_{i}$, with $c_{i}>0$, and $\sum_{1}^{n} c_{i}=1$, $x_{i}$ in $X_{0}$, together with the fact that $c x$ is a linear continuous transformation on $\mathfrak{X}$ to $\mathfrak{X}$ for fixed $c$. Hence, Price defines a convexification transformation $C^{*}$ on $X_{0}$ to $\mathfrak{X}$ by the expression $\sum_{i} \tau_{i} x_{i}$ in terms of a class $T$ of finite subsets $t$ of $\mathfrak{T}$ satisfying the following conditions: (a) if $t=\left(\tau_{1}, \cdots, \tau_{n}\right)$, then $\sum_{1}^{n} \tau_{i}=I$ ( $I$ the identity transformation in $\mathfrak{T})$; (b) if $t_{1}=\left(\tau_{11}, \cdots, \tau_{1 n}\right)$ and $t_{2}=\left(\tau_{21}, \cdots, \tau_{2 m}\right)$ are in $T$, then $t=t_{1} t_{2}=\left(\tau_{1 i} \tau_{2 j}, i=1, \cdots, n ; j=1, \cdots, m\right)$ is in $T$; (c) there exists a constant $W$ such that if $t$ is in $T$ then $\left\|\sum_{1}^{n} \tau_{i} x_{i}\right\| \leqq W$ $\cdot \max \left\|x_{i}\right\|$. This last condition is a generalization of the $W$-variation of Gowurin. For the integral definition the convexification transformation $C^{*}$ is defined in terms of the measure transformation function $\tau(E)$. The class $T$ consists of the elements $t=\left[\left(\sum_{i=1}^{i=n} \tau\left(E_{i}\right)\right)^{-1} \tau\left(E_{j}\right) ; j=1, \cdots, n\right]$ and $t^{\prime}=\left[\tau\left(E_{j}\right)\left(\sum_{i=1}^{i=n} \tau\left(E_{i}\right)\right)^{-1}\right.$; $j=1, \cdots, n]$, the $E_{i}$ being disjoint in $\&$ and $\tau\left(\sum_{i} E_{i}\right) \neq 0$, together with the extension of this set of elements to make $T$ multiplicative in accordance with condition (b) above. Then the integration definition follows the Birkhoff line based on $\sum_{k} \tau\left(E_{k}\right) C^{*} x\left(E_{k}\right)$ with $x\left(E_{k}\right)$ the set 
of values of $x(s)$ for $s$ on $E_{k}$. Thus $x(s)$ is integrable if there exists a subdivision $\sigma_{0}$ of $\sigma$, such that $x(s)$ is bounded on $E_{k}, \sum_{\sigma_{0}} \tau\left(E_{k}\right) C^{*} x\left(E_{k}\right)$ is unconditionally convergent, and the greatest lower bound of the diameter of this type of expression for all $\sigma$ is zero.

10. Gelfand-Pettis integral. The realization that the totality of linear continuous functionals or forms on a LNC space transfers the onus of convergence and related properties from the space to the space of real numbers gives rise to a convenient set of weak properties. We shall denote by $x^{*}$ an element of the space $\mathfrak{X}^{*}$ of linear forms of $\mathfrak{X}$. As is well known $\mathfrak{X}^{*}$ is a linear space normed and complete if $\left\|x^{*}\right\|=\mathrm{LUB}\left[\left|x^{*}(x)\right|\right.$ for $\left.\|x\|=1\right]$. The expression $x^{*}(x)$ or $\left(x^{*}, x\right)$ is a bilinear form on $\left(\mathfrak{X}^{*}, \mathfrak{X}\right)$. We then have a sequence $x_{n}$ weakly convergent to $x$ if, for every $x^{*}$, the sequence of real numbers $\left(x^{*}, x_{n}\right)$ converges to $\left(x^{*}, x\right)$, the function $x(s)$ on $(a, b)$ to $\mathfrak{X}$ is weakly continuous if $\left(x^{*}, x(s)\right)$ is continuous for every $x^{*}$, the function $x(s)$ is weakly measurable if $\left(x^{*}, x(s)\right)$ is measurable for every $x^{*}, x(s)$ is weakly integrable on a measurable set $E$ if $\left(x^{*}, x(s)\right)$ is integrable on $E$ for every $x^{*}$, and there exists an $x(E)$ of $\mathfrak{X}$ such that $\int_{E}\left(x^{*}, x(s)\right) d s$ $=\left(x^{*}, x(E)\right)$. Then we define $\int_{E} x(s) d s=x(E) . x(s)$ is weakly integrable if for every measurable subset $E$ of $\notin, x(s)$ is integrable on $E$. This definition for integrability for $\mathfrak{S}$ the real interval $(a, b)$ was suggested by Gelfand [22] and extended by Pettis [52] to the case when $\subseteq$ is a general space with a class of measurable sets and completely additive measure function. Pettis also discusses the properties of the integral and its relation to other integral definitions.

Usually any weak property must be supplemented by additional conditions to insure a corresponding strong property in LNC spaces. So it turns out that a necessary and sufficient condition that a function $x(s)$ be measurable in the strong (or Bochner) sense is that $x(s)$ be weakly measurable and almost separately valued (i.e., by neglecting a suitable set of measure zero in $\subseteq$ the values $x(s)$ form a separable subset of $\mathfrak{X}) .{ }^{2}$ The fact that the Bochner and Birkhoff integrals are special cases of the Gelfand-Pettis integral is cleverly deduced from the fact that under this type of integration, the operation of integra-

2 An elegant proof of this fact in E. Hille, Colloquium Lectures (Functional analysis and semi-groups) p. 36 , centers in the fact that if a LNC space $\mathfrak{X}$ is separable, the sequence $\left\{x_{n}\right\}$ is dense in the space, and $x_{n}{ }^{*}$ are selected so that $\left\|x_{n}{ }^{*}\right\|=1$ and $x_{n}^{*}\left(x_{n}\right)=\left\|x_{n}\right\|$, then the forms $x_{n}^{*}(x)$ define an isometric linear transformation on $\mathfrak{X}$ to the space of bounded sequences. There results the measurability of $\|x(s)-x\|$ for each $x$ of $\mathfrak{X}$ as the least upper bound of the sequence of measurable functions $\left|\left(x_{n}{ }^{*},(x(s)-x)\right)\right|$. This suggests another definition of measurability, viz., $x(s)$ is measurable if the function $\|x(s)-x\|$ is measurable for every $x$ of $\mathfrak{X}$. (See G. B. Price $[54$, p. 25].) 
tion and linear continuous transformation on a LNC space $\mathfrak{X}$ to a LNC space $\mathfrak{Y}$ are commutative in an optimum sense, viz., that any integral reducing to Lebesgue integration if $\mathfrak{X}$ is the space of reals which has this commutative property must be Gelfand-Pettis integrable. If $x(s)$ is strongly measurable, then the Gelfand-Pettis integral reduces to and agrees with the Birkhoff integral as well as a second integral definition of Dunford [14] requiring a function $x(s)$ to be integrable on $\mathfrak{S}$ if there exists a sequence of finite-valued measurable functions $x_{n}(s)$ converging to $x(s)$ almost everywhere and such that $\lim _{n} \int_{E} x_{n}(s) d s$ exists for all measurable subsets $E$. This latter definition connects up with the well known theorem that if a sequence of real-valued Lebesgue integrable functions $f_{n}(s)$ converges to $f(s)$ almost everywhere, then $\lim _{n} \int_{E} f_{n}(s) d s=\int_{E} f(s) d s$ for all measurable sets $E$ if and only if the $\int_{E} f_{n}(s) d s$ are uniformly absolutely continuous. Incidentally then, the properties " $\int_{E} x_{n}(s) d s$ uniformly absolutely continuous" and " $\int_{E} x_{n}(s) d s$ convergent for all measurable $E$ " are not equivalent in this more general setting since the first of these properties would imply the Bochner integrability of an $x(s)$ approached by a finite-valued sequence $x_{n}(s)$, but the second would not.

If $\left(x^{*}, x(s)\right)$ is integrable for all $x^{*}$, then this expression defines a linear continuous transformation on the space $\mathfrak{X}^{*}$ to the space $L^{1}$ of Lebesgue integrable functions on $\mathfrak{S}$. (See Pettis $[\mathbf{5 2}$, p. 286].) Consequently, $\int_{E}\left(x^{*}, x(s) d s\right.$ is a linear continuous form on $\mathfrak{X}^{*}$ for every $E$, that is, there exists for each measurable $E$ an element $x^{* *}(E)$ of $\mathfrak{X}^{* *}$, the conjugate of $\mathfrak{X}^{*}$, such that $\int_{E}\left(x^{*}, x(s) d s=\left(x^{*}, x^{* *}(E)\right)\right.$. It is only when $x^{* *}(E)$ is an element of $\mathfrak{X}$ for every $E$ that we get the Gelfand-Pettis integral, which seems natural since we should like our integral values in the same space $\mathfrak{X}$ with which we started. If, however, one is not averse to finding one's integral values in an extended space (after all Lebesgue integration notions involve the ideas of extension), then with Dunford [16] one has integrability of $x(s)$ if $\left(x^{*}, x(s)\right)$ is integrable for every $x^{*}, \int x(s) d s$ being defined as the $x^{* *}$ indicated. Pettis [52, p. 293] shows that if $x(s)$ is also strongly measurable, then $x^{* *}$ lies in $\mathfrak{X}$ for each $E$ and the Dunford third integral becomes a Pettis integral. There is an alternative way of looking at the Dunford third integral suggested by Phillips [53, p. 130]. Any member of $\mathfrak{X}$ is also a member of $\mathfrak{X}^{* *}$. Consequently defining the integrability of $x(s)$ in terms of the integrability of $\left(x^{*}, x(s)\right)$ $=\left(x^{* *}(s), x^{*}\right)$ is a weak integrability, a stepping down from $\mathfrak{X}^{* *}$ in its properties, what one might call a subweak integrability.

11. Phillips-Rickart generalizations. In the case of a LNC space $\mathfrak{X}$, we have in addition to the topology introduced by the norm, the 
topology associated with weak convergence of linear forms $\left(x^{*}, x\right)$. These give rise to a topological space, a vicinity $V\left(x_{1}^{*}, \cdots, x_{n}^{*}\right.$; $\left.x_{0} ; \epsilon\right)$ being defined by the $x$ for which $\left|x_{i}^{*}\left(x-x_{0}\right)\right|<\epsilon ; i=1, \cdots, n$. For $\mathfrak{X}^{*}$ we have two types of weak convergence, that induced by $\mathfrak{X}$ and that by $\mathfrak{X}^{* *}$. Both of these are special cases of linear topological spaces, spaces which are linear and have a topology in which addition and multiplication by real numbers is continuous, and which are determined by a system of vicinities of the origin satisfying the Hausdorff postulates (see v. Neumann [48]; Kolmogoroff [37]). In addition, for elegant results, it is assumed that the vicinities are convex (if $x_{1}$ and $x_{2}$ belong to a vicinity, then so does $a x_{1}+(1-a) x_{2}$ for $0 \leqq a \leqq 1)$. In that case any vicinity determines, for all $x$, a pseudo norm $\|x\|_{V}$ satisfying the norm properties, excepting that $\|x\|_{V}=0$ does not necessarily imply $x=0$ (if $\|x\|_{V}=0$ implies $x=0$, then the linear topological space becomes a linear normed space with $\|x\|_{V}$ as norm). We shall call such a linear topological space with convex vicinities a LCT space.

The Phillips generalization of the integration problem [53] (see also Birkhoff, Ann. of Math. vol. 38 (1937) p. 51) consists in the first place in playing in LCT spaces which include the LN spaces and the weak convergence on normed spaces as used by Pettis. The properties of real-valued functions not being available in this setting, an adaptation of the method used by Birkhoff for defining integrability becomes desirable. Phillips notes that in this type of definition (as in fact in all Lebesgue integrability definitions), there is involved an iterated limit. First one defines the unconditional convergence of an infinite series $\sum_{i} x\left(s_{i}\right) m E_{i}$ for some subdivisions and then takes the limit of these sums as to subdivisions, i.e., $\lim _{\sigma} \lim _{\pi} \sum_{\pi} x\left(s_{i}\right) m E_{i}$, where $\sigma$ stands for a subdivision and $\pi$ a finite number of integers. One can, however, define an iterated limit without assuming the existence of the interior limit simply by replacing lim lim by lim lim. In the definition of integral this means that the approximating sums need be in a sense only approximately convergent or summable. Thus, according to Phillips, a sequence of elements $x_{n}$ of $\mathfrak{X}$ is unconditionally summable to $x$ relative to a vicinity $V$ if there exists a subset $\pi_{V}$ of the integers such that if $\pi \geqq \pi_{V}$, then $\left\|\sum_{\pi} x_{n}-x\right\|_{V}<1$. A similar definition can be given for unconditional summability of a sequence of subsets $X_{n}$ of $\mathfrak{X}$ to an element $x$. Given now a space $\mathfrak{S}$, a class $\&$ of measurable subsets $E$, a completely additive measure function $\alpha$ on $\mathbb{E}$ to $\mathfrak{A}$, a many-valued function $x(E)$ on $\mathbb{E}$ to $\mathfrak{X}$ (being for instance the totality of values of a point function $x(s)$ for $s$ on $E$ ), then $x(E)$ is integrable relative to $\alpha(E)$ if for every measurable $E$ 
of $\mathbb{E}$, there exists an element $J(x, E)$ of $\mathfrak{X}$ such that for every vicinity $V$, there exists a subdivision $\sigma$ of $E$ such that $\sum_{i} x\left(E_{i}\right) \alpha\left(E_{i}\right)$ is unconditionally summable to $J(x, E)$ relative to $V$. In order to prove existence theorems, it is necessary to impose completeness conditions relative to a Moore-Smith mode of convergence depending on the directed set defined by the product space $\mathfrak{B P}$ of vicinities $V$ and subsets $\pi$ of the integers. Most of the usual properties of Lebesgue integration carry over, including the interchangeability of continuous linear transformation on a LCT space to a LCT space and integration.

Rickart [55], following the ideas of Phillips, and generalizing the definitions of Burkill [9] and Kolmogoroff [36], drops the measure function $\alpha(E)$ and is concerned only with a set function, which may be many-valued, on "measurable" subsets to a LCT space $\mathfrak{X}$. Further he is willing to consider a multiple-valued integral. He defines two subsets $X_{1}$ and $X_{2}$ to be equal within a vicinity $V$ if $X_{1}$ is contained in $X_{2}+V$, and $X_{2}$ contained in $X_{1}+V$. Then a sequence of sets $X_{n}$ is unconditionally summable to a set $X$ within $V$, if there exists a subset of the integers $\pi_{V}$ such that if $\pi \geqq \pi_{V}$, then $\sum_{\pi} X_{n}$ and $X$ are equal within $V$. Then $x(E)$ is integrable on $E_{0}$ if there exists a subset $I\left(x, E_{0}\right)$ of $\mathfrak{X}$ such that $\lim _{\sigma} \sum_{\sigma} x(E)=I\left(x, E_{0}\right)$. This means that for every $V$, there exists a subdivision $\sigma_{V}$ of $E_{0}$ such that if $\sigma \geqq \sigma_{V}$, then $\sum_{\sigma} x\left(E_{i}\right)$ is unconditionally summable to $I\left(x, E_{0}\right)$ within $V$. The closure of the set $I\left(x, E_{0}\right)$ is the integral set of $x$ over $E_{0}$. The case where $I\left(x, E_{0}\right)$ reduces to a single element is, of course, of special interest, but many integration properties can be proved for the integral as a closed set of elements.

Rickart considers also the special case when $x(E)$ is a bilinear function on a LCT space $\mathfrak{V}$ and the class $\mathbb{E}$ of measurable subsets of $\mathfrak{S}$, viz., $x(E)=B(y ; E)$, generalizing the integral of Gowurin [26]. Of course, in a bilinear transformation on $\mathfrak{Y} \mathbb{Z}$ to $\mathfrak{X}$, if $y(s)$ is a point function on $\subseteq$ to $\mathfrak{Y}$, and $x$ a single-valued set function $z(E)$ on $\&$ to $\mathbb{Z}$, explicit mention of the $z$ function is not really required. Rickart assumes $B(y, E)$ to be single-valued linear in $y$ for each $E$, and completely additive in $E$ for each $y$. In addition, it is assumed that there exists a real number $a \geqq 1$, such that if $Y_{i}, i=1, \cdots, n$, are any subsets of $\mathfrak{Y}, E_{i}$ are disjoint, $E_{i}^{j}, j=1, \cdots, k_{i}$, constitute a finite subdivision of $E_{i}$, then $\sum_{i} B\left(Y_{i}, E_{i}\right) \leqq V$ implies $\sum_{i} \sum_{j} B\left(Y_{i}, E_{i}^{j}\right)<a V$. An integral definition following the lines of the Phillips definition can be set up for $B(y(E), E)$. If $y(E)$ is a contractive function of sets, i.e., if $E^{\prime} \leqq E^{\prime \prime}$ implies $y\left(E^{\prime}\right) \leqq y\left(E^{\prime \prime}\right)$, then this integral of $B(y, E)$ is a special case of the general definition. Rickart also shows that by a 
proper definition of $B(y, E)$, the integral definition of Price [54] is included in the above considerations as a special case.

\section{Generalization of Lebesgue integration Based on ORder}

The generalizations of Lebesgue integration which we have been considering have been only indirectly concerned with the Lebesgue postulates mentioned at the beginning of this address. They have centered more in the consequences of these postulates, the notions of measurable sets and measurable functions. More closely connected with Lebesgue postulates is the general integral of Daniell $[11 ; 12]$. In the Daniell considerations, the basic idea is that the Lebesgue integral can be considered as an extension of the Riemann integral, in that the Lebesgue integral, reducing to the Riemann integral for continuous functions, applies to a larger class of functions and on this larger class has more extensive properties.

12. The Daniell integral. Daniell assumes as a basic class of elements the class $\mathfrak{F}$ of all real finite-valued functions on a general range $\mathfrak{S}$. From $\mathfrak{F}$ we select a subset $F_{0}$ of functions $f$, which is linear and with $f$ contains also $|f|$. Under linearity, this is equivalent to the assumption that with $f_{1}$ and $f_{2}, f_{1} \cup f_{2}$, the greater of $f_{1}$ and $f_{2}$, and $f_{1} \cap f_{2}$, the lesser of $f_{1}$ and $f_{2}$, belong to $F_{0}$. In other words, $F_{0}$ is a vector or linear lattice subset of $\mathfrak{F}$. On $F_{0}$ there is defined an integral $I(f)$ to real numbers, assumed to be linear $(\mathrm{L})$ and positive $(\mathrm{P})$ on $F_{0}$, i.e., satisfying postulates (3) and (4) of Lebesgue. In addition, $I$ satisfies an equivalent of the convergence property (6) of Lebesgue $(\mathrm{Cv})$ : if $f_{n}$ is a monotonic nondecreasing sequence of functions of $F_{0}$ converging to the zero function, then $\lim _{n} I\left(f_{n}\right)=0$. Note that from postulates (L) and (P) it follows that if $\lim _{n} f_{n}=f$ relative uniformly as to $F_{0}$ (there exists a function $f_{0}$ of $F_{0}$ such that for every $e>0$, there exists an $n_{e}$ such that if $n>n_{e}$, then $\left.\left|f_{n}-f\right|<e\left|f_{0}\right|\right)$, then $\lim _{n} I\left(f_{n}\right)=I(f)$. There is also assumed a "Stieltjes" integral $S(f)$, which satisfies the linearity and convergence postulates but replaces the positive property by a boundedness property: there exists a monotone transformation $M$ on positive functions of $F_{0}$, such that if $f$ is in $F_{0}$ then $|S(f)| \leqq M(|f|)$. This is equivalent to assuming that for $S(f)$ there exist two linear positive integrals $I_{1}$ and $I_{2}$ such that for every $f$ of $F_{0}: S(f)=I_{1}(f)-I_{2}(f)$; or that $S(f)$ transforms a set bounded in $F_{0}$ into a bounded set of numbers. As a consequence, most of the theory centers on the $I$ integral, having the $(\mathrm{L})(\mathrm{P})(\mathrm{Cv})$ properties. Conceptually $F_{0}$ abstracts the class of continuous functions or the step functions on a closed interval $(a, b)$, and $I$ the usual Riemann integral. 
The objective is to obtain a larger class $\mathbb{R}$, a subset of $\mathfrak{F}$ and containing $F_{0}$, of "integrable" functions and an integral $I$ on $\&$ reducing to $I$ on $F_{0}$. The class $\&$ should be a linear lattice. The integral $I$ should be linear and positive and have the following convergence properties, suggested by Lebesgue integration: (a) if $\left\{h_{n}\right\}$ is a monotone nondecreasing sequence of $\mathbb{R}$, converging to $h$ in $\mathfrak{F}$, and if $I\left(h_{n}\right)$ is a bounded sequence, then $h$ is in $\&$ and $\lim _{n} I\left(h_{n}\right)=I(h)$; (b) if $h_{n}$ is a sequence belonging to $\&$ for which there exists a function $h_{0}$ of $\&$ such that $\left|h_{n}\right| \leqq\left|h_{0}\right|$ for all $n$, and converging to a function $h$ in $\mathfrak{F}$, then $h$ is in $\&$ and $\lim _{n} I\left(h_{n}\right)=I(h)$.

In the Daniell procedure there is introduced an auxiliary class of functions $F_{1}$, being the limits of monotone increasing sequences of functions of $F_{0}$, which may take $+\infty$ as values. If we denote functions of $F_{1}$ by $g$, then $I(g)$ is defined as $\lim _{n} I\left(f_{n}\right)$ where $f_{n}$ is any monotone increasing sequence in $F_{0}$ converging to $g$, and may have $+\infty$ as value. $F_{1}$ is closed under extension by monotone increasing sequences. For elements of $\mathfrak{F}$ we now define an upper integral by the condition: if $h$ is in $\mathfrak{F}$ and no function of $F_{1}$ dominates $h$, then $\bar{I}(h)=+\infty$; otherwise $\bar{I}(h)=\mathrm{GLB}\left[I(g)\right.$ for all $g$ of $\left.F_{1} \geqq h\right],+\infty$ being a permissible value. It might be noted here that the subsidiary class $F_{1}$ could be omitted from this definition if we defined $\bar{I}(h)=\mathrm{GLB}\left[\lim _{n} I\left(f_{n}\right)\right.$ for all monotone increasing sequences $\left\{f_{n}\right\}$ from $F_{0}$ such that $\left.\lim _{n} f_{n} \geqq h\right]$. This form bears a close relationship to the definition of upper measure of a set $E$ as the greatest lower bound of the sums of the lengths of intervals covering $E$.

This upper integral has the usual properties, viz., (a) $\bar{I}\left(h_{1}+h_{2}\right)$ $\leqq \bar{I}\left(h_{1}\right)+\bar{I}\left(h_{2}\right) ;(\mathrm{b}) \bar{I}(c h)=c \bar{I}(h)$ if $c \geqq 0 ;(c)$ if $h_{n} \geqq 0$, and $0 \leqq h \leqq \sum_{n} h_{n}$, then $\bar{I}(h) \leqq \sum_{n} \bar{I}\left(h_{n}\right)$. A lower integral is defined by $-\bar{I}(-h)$, and now a function $h$ in $\mathfrak{F}$ is said to be integrable and in class $\&$ if $\bar{I}(h)$ $=-\bar{I}(-h)$ with $\bar{I}(h)$ finite, the value of the integral $\bar{I}(h)$ being the common value. The $(\mathrm{Cv})$ property of $I$ on $F_{0}$ guarantees that if $f$ is in $F_{0}$ it is also in $\&$, the value of $I(f)$ being unchanged. This class of functions $\&$ has the Lebesgue properties mentioned above. Moreover, it is possible to prove that the class $F_{0}$ is dense in $\&$, in the sense that $h$ belongs to $\&$ if and only if for every $e>0$ there exists an $f_{e}$ in $F_{0}$ such that $\bar{I}\left(\left|h-f_{e}\right|\right)<e$. Moreover, if the class $\&$ is suitably extended to include functions assuming $\pm \infty$ as values, then this extended class can be shown to be complete under the metric $I\left(\left|h_{1}-h_{2}\right|\right)$.

Banach [1] has given another approach to the Daniell type of integral generalization. $F_{0}$ is again a linear lattice subset of the space $\mathfrak{F}$ of finite-valued functions on $\mathfrak{S}, I$ is linear and positive on $F_{0}$, but the convergence condition $(\mathrm{Cv})$ is replaced by one suggested by the 
Osgood theorem or the convergence property (b) of Lebesgue integrals, viz., if the sequence $f_{n}$ is bounded in $F_{0}$ and converges to zero, then $\lim _{n} I\left(f_{n}\right)=0$. This is equivalent to: if $f_{n}$ is bounded below in $F_{0}$ and $\lim _{n} f_{n} \geqq 0$, then $\lim _{n} I\left(f_{n}\right) \geqq 0$. It is possible to show that these conditions on $I$ are equivalent to those of Daniell, though to deduce the Banach condition $(\mathrm{Cv})$ from the Daniell ones the extension theory may be needed. The definition of upper and lower integrals is applied to a subset $H$ of functions of $\mathfrak{F}, H$ being the class of functions $h(s)$ for which there exist sequences of functions $f_{n}^{\prime}$ and $f_{n}^{\prime \prime}$ of $F_{0}$ such that

$$
\varlimsup_{n} f_{n}^{\prime} \leqq h \leqq \underline{\lim }_{n} f_{n}^{\prime \prime}
$$

It is easy to show that the class $H$ agrees with the functions $h$ of $\mathfrak{F}$ for which in the Daniell formulation there exists a function $g_{1}$ of $F_{1}$ and a function $g_{2}$ with $-g_{2}$ in $F_{1}$ such that $g_{2} \leqq h \leqq g_{1}$. For functions $h$ of $H, \bar{I}(h)$ is the greatest lower bound of $\lim _{n} I\left(f_{n}\right)$ for all sequences $f_{n}$ bounded below in $F_{0}$ such that $\underline{\lim }_{n} f_{n} \geqq h$; the definition of a lower integral follows the complementary procedure. The ultimate class $\&$ of integrable functions for which the upper and lower integrals are equal to the same finite number agrees with the class $\mathfrak{R}$ as defined by Daniell. A procedure similar to that of Banach is presented by Goldstine [23]. It hardly deserves mention that the Daniell procedure centering on monotone sequences is conceptually simpler than the Banach procedure involving lim and $\overline{\mathrm{lim}}$.

[Since this address was given another approach to the Daniell integral has been given by $M$. H. Stone $[61, I]$. The only change in the postulates on $F_{0}$ and the integral $I$ on $F_{0}$ is in the convergence postulate $(\mathrm{Cv})$ which in the Stone version reads: If $f_{n}$ and $f$ belong to $F_{0}$ and $|f| \leqq \sum_{n}\left|f_{n}\right|$, then $|I(f)| \leqq \sum_{n} I\left(\left|f_{n}\right|\right)$. These postulates are actually equivalent to the Daniell set if the latter's $(\mathrm{Cv})$ postulate is stated in the form: If $f_{n}$ is any monotonic increasing sequence from $F_{0}$ converging to $f$ in $F_{0}$, then $\lim _{n} I\left(f_{n}\right)=I(f)$. Stone does not introduce the class $F_{1}$ but defines an upper integral for positive functions $h$ in $\mathfrak{F}$ by the condition $\bar{I}(h)$ is the greatest lower bound of $\sum_{n} I\left(f_{n}\right)$ for all sequences $f_{n} \geqq 0$ from $F_{0}$ such that $\sum_{n} f_{n} \geqq h$. Since under these conditions $\sum_{n} f_{n}$ belongs to $F_{1}$, it is obvious that the upper integrals for positive-valued functions for Stone and Daniell agree. The interesting new thing is that if $\mathfrak{F}$ is extended to include functions $\bar{h}$ having $+\infty$ as values and $\bar{I}(\bar{h})$ is defined as above, for positive functions, then $\bar{I}(|\bar{h}|)<\infty$ only if $\bar{h}$ differs from an $h$ in $\mathfrak{F}$ by a null function, one for which $\bar{I}(|\bar{h}-h|)=0$. Then $\bar{h}-h$ vanishes excepting on a null set $E$, one for which $\bar{I}\left(\chi_{E}\right)=0$ where $\chi_{E}$ is the char- 
acteristic function of $E$. Further, it develops that the space for which $\bar{I}(\bar{h})<\infty$ is a linear normed complete space with $\bar{I}(|\bar{h}|)$ as norm. The space of $L$-integrable functions is then the completion of the space $F_{0}$ under the norm $I(|f|)$. This is reminiscent of the definition of Lebesgue integration due to Dunford [15].

A more general approach to an integral of the Daniell type has been suggested by the Bourbaki group via Stone [61, IV] and independently by McShane [43]. The principal change is in the class $F_{1}$ of limits of monotone increasing sequences $f_{n}$. The sequence is replaced by a generalized monotonic or directed set, such a set being assumed to be monotone according to the order relation "ฏ” denoted by $\Delta$ if for any two functions $f_{1}$ and $f_{2}$ of the set there exists a function $f_{3}$ in the set dominating $f_{1}$ and $f_{2}: f_{3} \geqq f_{1}, f_{3} \geqq f_{2}$. Obviously, the least upper bound of such a directed set agrees with its Moore-Smith limit with $\Delta$ (or $\leqq$ ) as the order relation. The convergence postulate $(\mathrm{Cv})$ is now strengthened to apply to $\Delta$-monotone sets, i.e., if $\left\{f_{\Delta}\right\}$ is a $\Delta$-monotone set in $F_{0}$ converging to an $f$ in $F_{0}$, then $\lim _{\Delta} I\left(f_{\Delta}\right)=I(f)$. The class $F_{1}$ of Daniell is replaced by the class $F_{1}^{\prime}$ of limits of $\Delta$ monotone sets of functions of $F_{0}$, and the upper integral defined in terms of functions of $F_{1}^{\prime}$. The difference between the class of functions integrable by the Daniell-Stone sequential procedure and by $\Delta$ monotone sets is a matter of null functions in the sense that if $h$ belongs to $\Omega_{\Delta}$, then $h=h_{\sigma}+h_{\nu}$, where $h_{\sigma}$ is a sequential $\&$ function and $\bar{I}_{\Delta}\left(\left|h_{v}\right|\right)=0$.]

13. Measurability of functions and sets in Daniell integrals. In the case of Lebesgue integrals, measurability of functions is tied up with measurable sets. Since for Daniell integrals sets play no role, measurability must be characterized in terms of integrability. We note that for Lebesgue integrals, integrability and measurability are equivalent for bounded functions on a closed interval; for unbounded functions, measurability is equivalent to the integrability of every truncated part of a function, i.e., $f$ is measurable if for every $a \leqq b$ the function $f_{a b}=a \cup f \cap b$ (equal to $a$ for $f \leqq a$, equal to $f$ for $a \leqq f \leqq b$, equal to $b$ for $f \geqq b)$ is integrable. Since in the case of Daniell integrability it is not assured that $f \equiv 1$ is integrable (Daniell purposely avoided this) we get measurability relative to any positive integrable finite-valued function $h, f$ being measurable relative to $h$ if for all $a \leqq b$ the function $f_{a b}=a h \cup f \cap b h$ is integrable. Daniell $[12$, p. 210] shows that if a function $f \geqq 0$ is measurable relative to $h$, if $I(f)<\infty$, and there exists a finite-valued function $\theta$ such that $f=\theta h$, then $f$ is integrable. [Stone $[61$, II] defines a function $f$ to be measurable in case for any two integrable functions $h_{1}$ and $h_{2}$, the median function of $f, h_{1}$ and $h_{2}$ is 
integrable, the median of three numbers $a, b, c$ being obviously the number between the two others, in symbols $U[a \cap b, a \cap c, b \cap c]$ $=\cap[a \cup b, a \cup c, b \cup c]$. Then any integrable function is measurable. Obviously, a function, in order to be measurable in the Stone sense must be measurable relative to every positive integrable function in the Daniell sense. The converse is also true.]

For measurable sets there are various modes of procedure, each centering in the characteristic function $\chi_{E}$ of the set. On the one hand (a) we can say that $E$ is measurable if the corresponding characteristic function is integrable; or (b) we can say that $E$ is measurable if $\chi_{E}$ is measurable; again, we can observe (c) that if for every $E$ we consider the upper integral $\bar{I}\left(\chi_{E}\right)$, we have defined on $E$ a Carathéodory upper measure, which gives rise to a class of measurable sets via the usual condition: $M$ is measurable if and only if for all $E, \mu^{*} E=\mu^{*} E M+\mu^{*}(E-E M)$, where $\mu^{*}$ is the upper measure, and $E M$ is the product of the sets $E$ and $M$. Obviously, the class of measurable sets may prove quite trivial. In case the unit function $h \equiv 1$ is integrable, Goldstine [23] has shown that a function $h$ is integrable if and only if its integral is expressible in the form $\int h(s) d \mu$, where $\mu$ is the Carathéodory measure function defined by $\bar{I}\left(\chi_{E}\right)$. [Stone [61, II] has shown that measurability, as defined by him, of the unit function $h \equiv 1$ is a necessary and sufficient condition for the equivalence of measurability of a set $E$ according to (b) and (c) above. In that case it is also possible to show that a finite-valued function is measurable if and only if the sets $E[a \leqq f \leqq b]$ are measurable for all $a<b$, and the integral of an integrable function can be defined after the Lebesgue manner.]

14. Extensions of Daniell integrals. An examination of the Daniell procedure reveals the fact that the real-valued functions on a general set $\mathfrak{S}$, for which an integral is sought, could be replaced by functions to more general spaces, having the linearity and order properties of the real numbers. The most convenient such spaces are linear partially ordered and lattice spaces $\mathfrak{X}$. A linear partially ordered space is one in which an order $\geqq$ is defined between some elements of the space, subject to the conditions (a) if $x_{1} \geqq x_{2}$, then for every $x: x_{1}+x$ $\geqq x_{2}+x$, (b) $a \geqq 0$ and $x \geqq 0$ imply $a x \geqq 0$. The space $\mathfrak{X}$ is a lattice if for each $x_{1}, x_{2}$ the greatest lower bound $x_{1} \cap x_{2}$ and the least upper bound $x_{1} \cup x_{2}$ exist in the space; $\mathfrak{X}$ is a $\sigma$-complete linear lattice if any sequence $x_{n}$ bounded above (bounded below) has a least upper bound (greatest lower bound), a complete lattice if sequence is replaced by set. (See Kantorovic [34] and Birkhoff [5, p. 238].) If we assume $\mathfrak{F}$ to be the set of all functions on a general set $\mathfrak{S}$ to a linear complete 
lattice $\mathfrak{X}, F_{0}$ to be a linear sublattice of $\mathfrak{F}, I$ on $F_{0}$ to real numbers, linear, positive and having the $(\mathrm{Cv})$ property of Daniell, then the Daniell extension theory is possible on elements of $\mathfrak{F}$, a Lebesgue type of integral exists having the Lebesgue convergence properties on a subset $\mathfrak{R}$ of $\mathfrak{F}$ of "integrable" functions, convergence of the functions being of the order type. (Cf. Birkhoff [5, pp. $59 \mathrm{ff}$.$] .) By a$ proper treatment of functions taking on "extended" values it would seem to be possible to assert the completeness of the space of integrable functions under the norm $I(|h|)$, where $|h|=h^{+}+h^{-}$, with $h^{+}=h \cup 0$ and $h^{-}=-h \cup 0$. The theory indicated has been carried through by M. Orihara and G. Sunouchi [51].

From these considerations it is obviously a simple matter to drop the idea that $\mathfrak{F}$ is a class of functions and replace it by a set of elements having an adequate number of the properties of the class of all finite real-valued functions on a general range. Such a set of elements is a linear $\sigma$-complete lattice. Then $F_{0}$ is again a linear sublattice of $\mathfrak{F}, I$ is a linear positive form on $F_{0}$ to real numbers, having the convergence property $(\mathrm{Cv}):$ if $f_{n}$ of $F_{0}$ is a monotone decreasing sequence such that $\bigcap_{n} f_{n}=0$, then $\lim _{n} I\left(f_{n}\right)=0$. A "Stieltjes" integral $S$ would be linear, have the $(\mathrm{Cv})$ property, but replace the positive property by a boundedness condition, e.g., if $F_{0}^{\prime}$ is a bounded subset of $F_{0}$, then the set of numbers $S\left(F_{0}^{\prime}\right)$ is bounded. The class of functions $F_{1}$ used in the definition of upper integral can be by-passed by defining the upper integral $\bar{I}(h)$ as the greatest lower bound of $\lim _{n} I\left(f_{n}\right)$ for all monotone sequences $f_{n}$ chosen from $F_{0}$ for which $\mathrm{U}_{n}\left(h \cap f_{n}\right)=h$. If no such sequence exists, then $I(h)=+\infty$. It is possible to introduce a parallel to the class $F_{1}$ of Daniell which may include functions having $+\infty$ as a value. We simply assume $F_{1}$ to consist of all monotone nondecreasing sequences in $F_{0}$. Two such sequences $f_{1 n}, f_{2 m}$ define the same element if $\bigcup_{n}\left(f_{1 n} \cap f_{2 m}\right)=f_{2 m}$ for every $m$ and $U_{m}\left(f_{1 n} \cap f_{2 m}\right)=f_{1 n}$ for every $n$. If a sequence has a least upper bound in $\mathfrak{F}$, the sequence is equivalent to this element. The definition of order in $F_{1}$ and relative to $\mathfrak{F}$ is obvious, as is the extension of the integral $I$ to $F_{1}$. It can be shown that the upper integral $\bar{I}$ has the usual properties. This also holds for the resulting $I$ integral for which $\bar{I}(h)=-\bar{I}(-h)$ finite, at least as applied to elements in $\mathfrak{F}$. There is, however, some difficulty about the completeness of the class of integrable functions since it is not possible to speak of a "null" set, only of null elements for which $\bar{I}(|h|)=0$. Izumi and Nakamura [32, III] have suggested the above extension process based on the postulates and procedure of Banach [1]. Matsuyama [44] has followed the Goldstine [23] presentation and given more detail. Reference should also be made to Nakano [47, II]. 
The next obvious generalization is to replace the value space of the integral $I$ on $F_{0}$ by a more general space, having the real numbers as a special case, for instance, by a linear lattice $\mathfrak{V}$ assumed to be complete. The $I$-integral then becomes a linear order preserving transformation satisfying the $(\mathrm{Cv})$ postulate. The $S$-integral replaces the order preserving condition either by a boundedness condition or equivalently assumes it to be the difference of two positive $I$-transformations. An upper integral is definable on elements of $\mathfrak{F}$ and selects from $\mathfrak{F}$ a linear subclass by the condition that $\bar{I}(h)$ be in $\mathfrak{Y}$. It has the properties $\bar{I}(a h)=a \bar{I}(h)$ for $a \geqq 0$, and $\bar{I}\left(h_{1}+h_{2}\right) \leqq \bar{I}\left(h_{1}\right)+\bar{I}\left(h_{2}\right)$, but there is doubt whether the convergence property: if $h_{n} \geqq 0$, and $0 \leqq h \leqq \sum_{n} h_{n}$, then $\bar{I}(h) \leqq \sum_{n} \bar{I}\left(h_{n}\right)$ holds. For the class of integrable functions for which $\bar{I}(h)=-\bar{I}(-h)$, it is possible to assert linearity, but the Lebesgue convergence properties may not be valid. We might, however, note that the Hahn-Banach extension theorem [3, p. 28] on linear order preserving transformations on a $\sigma$-complete linear lattice $\mathfrak{X}$ to a complete linear lattice $\mathfrak{Y}$ is valid. [See Izumi, Matuyama, and Orihara [32, V].] Hence, it would be possible to extend the linear order preserving integral $I$ on $F_{0}$ to a linear order preserving integral on the linear subset $\bar{F}$ of $\mathfrak{F}$ for which $\bar{I}(\bar{h})$ is in $\mathfrak{Y}$, and satisfying the condition $I(\bar{h}) \leqq \bar{I}(\bar{h})$ for all $\bar{h}$ in $\bar{F}$. This would be uniquely determined on the set of $\bar{h}$ 's for which $\bar{I}(\bar{h})=-\bar{I}(-\bar{h})$.

15. Special cases. The Daniell integral theory, considering the integral as an order preserving transformation on a linear lattice to a linear lattice, assumes that the "integral" on the basic set $F_{0}$ is given. Bochner and Fan [7] develop such an integral in connection with the problem of determining the most general linear order preserving transformation on the space of continuous functions on a finite interval to a linear partially ordered monotonely $\sigma$-complete space $\mathfrak{X}$ (if $x_{n}$ is a monotone increasing sequence bounded in $\mathfrak{X}$, then $U_{n} x_{n}$ $=\lim _{n} x_{n}$ exists in $\left.\mathfrak{X}\right)$. They find that such a transformation takes the form of $\int f(s) \alpha(d I)$, where $\alpha(I)$ is a non-negative additive interval function on $(a, b)$ to $\mathfrak{X}$, the integration being the order limit in the sense of successive subdivisions of $(a, b)$. The generalization to order preserving linear transformations on the set of all bounded realvalued functions on a general space $\subseteq$ is easily made, the result being $T(f)=\int f(s) \alpha(d E)$ where $\alpha(E)$ is a non-negative additive set function on all subsets $E$ of $\subseteq$ to $\mathfrak{X}$, and $\mathfrak{X}$ is a linear partially ordered space with a Moore-Smith monotone complete property (if $x_{p}$ is a directed set monotone increasing in $p$, and bounded in $\mathfrak{X}$, then the least upper bound of $x_{p}$ exists in $\left.\mathfrak{X}\right)$. The integral can be defined as the common value of $\bigcap_{\sigma} \sum_{\sigma} M_{i} \alpha\left(E_{i}\right)$ and $U_{\sigma} \sum_{\sigma} m_{i} \alpha\left(E_{i}\right)$, where $M_{i}$ 
and $m_{i}$ are respectively the least upper bound and greatest lower bound of $f$ on $E_{i}$ and $\sigma=\left(E_{1}, \cdots, E_{n}\right)$ stands for any subdivision of $\Im$ into a finite number of disjoint sets.

These special instances suggest that it should be possible to work out theories of integration paralleling to some extent that on normed linear spaces. We could consider the case where the integrand is of the form $f(s) \alpha(E), f$ real-valued on a general set $\mathfrak{S}, \alpha(E)$ defined on "measurable" subsets of $\subseteq$ to a linear partially ordered, or linear lattice space with proper completeness properties, $\alpha(E)$ either finitely additive or if completely additive then in the sense of unconditional convergence. We might note that if for a real-valued function $f(s)$ we define the function of intervals $\alpha\left(t_{1}, t_{2}\right)=\chi_{E}(s)$, where $E=\left[s \mid t_{1} \leqq f<t_{2}\right]$, then $\int_{-\infty}^{\infty} t d \alpha(I)$ is such an integral and reproduces the function $f$. The Lebesgue integral, when it exists, permits one to interchange integrations: $\int f=\int_{-\infty}^{\infty} t d \int \alpha(I)$. The resolution of the identity for bounded Hermitian transformations in Hilbert space is another such instance.

The complementary situation where $f$ is on $\subseteq$ to $\mathfrak{X}$, a linear partially ordered or lattice space and $\alpha(E)$ a real-valued function on certain subsets of $\mathfrak{S}$ with proper additive properties, is obviously possible also. Izumi [32, VII] following Bochner's integration theory on LNC spaces has carried through this type of definition assuming $\subseteq$ to be the linear interval $(a, b)$. He also points out that the functions on $\mathfrak{S}$ could be replaced by a linear $\sigma$-complete lattice $\mathfrak{B}$ with a sublattice $V_{0}$ (step functions) on which $I$ is defined with values in a $\sigma$-complete lattice $\mathfrak{V}$. The extension of $I$ to a larger subset of $\mathfrak{B}$ follows the extension method suggested by MacNeille [41], an element $f$ of $\mathfrak{B}$ being integrable if there exists a sequence of positive elements of $V_{0}: u_{n}$ such that $f=\sum_{n} u_{n}$ and $\sum_{n} I\left(u_{n}\right)$ converges in the order sense.

16. Carathéodory theory of integrals over spaces without points. Most of the integrals we have been considering operate on functions, but are functions on a general class of elements. In particular measure functions are on certain classes of subsets of a given class. In this connection there is an obvious suggestion to replace the subsets of a given space by a class of elements, which has some of the properties of the class of all subsets of a given class. This suggestion was made by Carathéodory [10] and expanded by others, e.g., Wecken [64], Ridder [57], Olmsted [50], and A. Pereira Gomes [24].

We assume a basic class $\&$ of elements $E$, which form a Boolean algebra, having a null element 0 , an all element 1 , addition or join, multiplication or meet, and a complementing process. If the sum or join of any sequence of elements exists, we shall call $\sigma$-complete. If the join of any set of elements exists, we shall call $\&$ complete. A 
set of "measurable" sets then would correspond to a $\sigma$-complete subalgebra containing the null and all element. Obviously various combinations are possible as in set theory.

A Carathéodory upper measure function $\mu^{*}$ on $\&$ satisfies the conditions (a) $0 \leqq \mu^{*} E \leqq \infty$; (b) $\mu^{*}\left(E_{1} \cup E_{2}\right) \leqq \mu^{*} E_{1}+\mu^{*} E_{2}$; (c) if $E \leqq \bigcup_{n} E_{n}$ (which if $U_{n} E_{n}$ does not exist might be replaced by the condition $\left.E=U_{n}\left(E_{n} \cap E\right)\right)$, then $\mu^{*} E \leqq \sum_{n} \mu^{*} E_{n}$. A class of measurable elements $M$ would be defined by the condition $\mu^{*} E=\mu^{*} E \cap M+(E \cap C M), C M$ complementary of $M$, for all $E$ of $\leftleftarrows$. Such an outer measure function can be deduced from any non-negative function $p(E)$ on with $\mu^{*} E$ the greatest lower bound of $\sum_{n} p\left(E_{n}\right)$ for all $U_{n} E_{n} \geqq E$, which is similar to the Daniell definition of upper integral. The resulting measure function and class of measurable sets may be trivial. Usually one postulates a set $\mathfrak{M}$ of measurable elements $M$ and a completely additive measure function on $\mathfrak{M}$, with the additional condition that there exists a sequence of elements $M_{n}$ in $\mathfrak{M}$ such $\mu\left(M_{n}\right)<\infty$ for all $n$ and $\mathrm{U}_{n} M_{n}=1$.

One is faced with the problem of defining a "point" function in spite of the fact that no "point" elements are postulated in $\&$. For bounded functions, Carathéodory notes that in a sense a "point" function is determined by its least upper bound and greatest lower bound on the sets to which the "point" belongs. He therefore assumes that a point function is determined by two functions $\alpha, \beta$ on $\&$ to real numbers, $\alpha$ decreasing and $\beta$ increasing. There is also assumed a dense sequence of values $\left\{y_{n}\right\}$ on the finite interval to which $\alpha$ and $\beta$ are defined and a corresponding sequence of elements $E_{n}$ such that $\beta\left(E_{n}\right) \leqq y_{n}$ and for every element $E$ such that $E \cap E_{n}=0$ we have $\alpha(E) \geqq y_{n}$. This definition is conceptually rather complicated, due perhaps to the desire of defining a "point" function in terms of realvalued set functions. Another somewhat simpler approach to the Carathéodory type of point function (function on $\&$ to real numbers) has been given by Kappos [35]. Kappos defines first simple functions (essentially denumerably-valued step functions) and obtains a general point function as a Dedekind cut in this class of functions which constitutes a linear lattice. The process of defining a point function is much simplified if we reverse our desires and define a point function as a function on the reals $-\infty<y<\infty$ to elements of (₹. We note, for instance, that for a real-valued point function the sets (a) $E_{y}=E[f(s)$ $=y]$; (b) $E_{y}=E[f(s) \geqq y] ;$ (c) $E_{y}=E[f(s)>y]$; (d) $E_{y}=[f(s) \leqq y]$; and (e) $E_{y}=[f(s)<y]$, defining functions on $y$ to $(E$, all determine the point function $f$. Corresponding to (a) we would define a function $E_{y}$ on $-\infty<y<\infty$ a single-valued point function if it satisfies the 
additional conditions (1) $U_{y} E_{y}=1$, (2) $y_{1} \neq y_{2}$ implies $E_{y_{1}} \cap E_{y_{2}}=0$. Condition (b) would require $E_{y}$ to satisfy the following additional conditions (1) $\bigcup_{y} E_{y}=1, \cap_{y} E_{y}=0$, (2) $y_{1} \leqq y_{2}$ implies $E_{y_{1}} \geqq E_{y_{2}}$, (3) $\cap_{y<y_{1}} E_{y}=E_{y_{1}}$ or $E_{y-0}=E_{y}$ for all $y$. There are corresponding obvious conditions for the forms (c), (d), (e). The product involved in (3) exists if $E$ is $\sigma$-complete, because of the monotonic assumption (2). If $\&$ is complete, it is obvious that one can define a point function of type (b) from one of type (a). Point functions of type (b) with the basic class forming a $\sigma$-complete Boolean algebra are usually taken as a basis for study. See, e.g., Olmsted [50]. Obviously, such a function is completely determined by its values at a denumerably dense set on $-\infty<y<\infty$. By following through the methods used in proving that the ordinary class of measurable functions is a linear $\sigma$-complete lattice, it is possible to show that the set of all point functions form such a linear $\sigma$-complete lattice, but even more that they form a ring of functions (one easily defines $f^{2}$; then $4 f g=(f+g)^{2}$ $\left.-(f-g)^{2}\right)$. The road to integrability is obvious. If $\mathfrak{M}$ is the class of "measurable" elements $M$ with the completely additive measure function $\mu M$, and $E_{y}$ is a point function on $(-\infty, \infty)$ to $\&$, then $E_{y}$ is integrable if and only if $\int_{-\infty}^{\infty} y d \mu\left(E_{y}\right)$ exists.

It is possible to define a "characteristic" point function of an element $E$ of $E$ by the condition $\chi(E, y)=1$ if $y \leqq 0, \chi(E, y)=E$ if $0<y \leqq 1 ; \chi(E, y)=0$ if $y>1$. These characteristic functions form a Boolean algebra of the same type as (E. Moreover, it is possible if (E) is $\sigma$-complete to express any point function in the form $\int_{-\infty}^{\infty} y d \chi\left(E_{y}\right)$, where the integral is defined after the manner of Lebesgue, first for bounded functions giving $\int_{a}^{b} y d \chi\left(E_{y}\right)$ when $E_{y}=0$ for $y<a$, and $E_{y}=1$ for $y>b$, and then as $\lim _{n} \int_{-n}^{n} y d \chi\left(E_{y}\right)$ if $E_{y}$ ranges over the entire infinite interval, i.e., is unbounded. See Birkhoff $[5$, p. 251]. The linear lattice of all point functions is consequently determined by the Boolean algebra of characteristic functions via an integration process.

An important result of the latter type is due to Freudenthal [21]. Let $\mathfrak{X}$ be a $\sigma$-complete linear lattice. Assume that $\mathfrak{X}$ contains a unit $u$, an element of $\mathfrak{X}$ satisfying the conditions (a) $u \geqq 0$, (b) $u \cap|x|=0$ implies $x=0$. Then the subset of $\mathfrak{X}$ which consists of the elements $e$ such that $e \cap(u-e)=0$ form a $\sigma$-complete Boolean algebra $(5 . T h e n$ to every element $x$ of $\mathfrak{X}$ there corresponds a "point" function $e_{y}$ on $-\infty<y<\infty$ to $\mathbb{E}^{-}$such that $x=\int_{-\infty}^{\infty} y d\left(e_{y}\right)$.

17. An additional approach to abstract integrals. Izumi [32, IV] has suggested another aspect of ordinary integration from which the 
notion of abstract integration might be considered. In defining the Riemann integral of a function $f(s)$ on $a \leqq s \leqq b$, we consider the approximating sums $\sum_{i} f\left(s_{i}^{\prime}\right)\left(s_{i}-s_{i-1}\right)$ for a subdivision $\sigma$ of $(a, b)$. Considered from the point of view of linear forms we have defined for every $\sigma$ a set of linear forms $I_{\sigma}(f)$ valid for all finite-valued functions on $(a, b)$. The subdivisions $\sigma$ form a directed set by inclusion. The class of Riemann integrable functions is that for which $\lim _{\sigma} I_{\sigma}(f)$ exists. This leads to the following abstraction. Given a linear space $\mathfrak{X}$ of elements $x$, and a set of linear forms $L_{\alpha}(x)$ on $\mathfrak{X}$ to real numbers. Assume that the $\alpha$ form a directed set to which a Moore-Smith limit is applicable. Then the space of "integrable" elements is that subset $X_{0}$ of $\mathfrak{X}$ for which $\lim _{\alpha} L_{\alpha}(x)$ exists, and the integral $L(x)$ is the limit. Because of the linearity property of the limit notion, it follows at once that $X_{0}$ is linear and $L$ is linear on $X_{0}$. Additional conditions on $X$ and the forms $L_{\alpha}(x)$ must be added to obtain properties of $L(x)$, similar to those of Riemann and Lebesgue integration. Obviously, if $\varlimsup_{\alpha} L_{\alpha} x$ exists as a finite number for all $x$ of $\mathfrak{X}$, then this defines a semi-additive function $\bar{L}(x)$, i.e. $\bar{L}\left(x_{1}+x_{2}\right) \leqq \bar{L}\left(x_{1}\right)+\bar{L}\left(x_{2}\right)$, and $\bar{L}(a x)=a \bar{L}(x)$ for $a \geqq 0$, making the Hahn-Banach theorem applicable, and assuring us of the existence of a linear form $L(x)$ on all $X$ which can be regarded as an extension of $L(x)$ on $X_{0}$. Extensions of these considerations when the real number system as the values of the linear forms are replaced by linear normed or topological spaces, or linear lattices, are possible.

I must bring this meager survey of the development of abstract integration to a close. I feel apologetic about not including many important phases such as the generalization of the Radon-Nikodym theorem [49] starting with the Riesz paper on Sur quelques notions fondamentales dans la thêorie des opérations lineaires [59] and effectively continued by Dieudonné [13]. Other points would be derivative properties, Denjoy types of integrals, and integrals of nonlinear type. If there is a unifying thread running through this survey, it would be that integration is essentially a linear process operating on a linear space. As suggested by the Lebesgue postulates, one abstracts and considers integration as a transformation from one space to another preserving certain properties, such as linearity, order, boundedness, and convergence. Of course, one could make the rash statement that an integral is a linear "continuous" form, a statement which is quite in keeping with recent trends (A. Weil, L. Schwartz). This is a logical sequel to the expression of the most general linear form on continuous functions on a finite interval as a Stieltjes integral or that on the space of bounded functions as a generalized 
integral. A second notion which plays a prominent role is that of extension. The value of an "integral" is known for a certain group of elements, subset of a larger group. One tries to extend to a larger group preserving certain properties, obtaining other properties for the larger group. For instance, the completion procedures of the rational numbers to the real number systems may be usable. There is, of course, the question of what limitations one should put on the notion of extension. Thus, for instance, the space of all linear forms on a linear space forms again a linear space, the first conjugate of the original space. The second conjugate includes the original space as a subset, which might be considered as an extension of the original linear space. In case the original space is not reflexive, the process can continue. Where stop? Perhaps this is a good place to conclude.

\section{BIBLIOGRAPHY}

1. S. Banach, The Lebesgue integral in abstract spaces, Saks, Theory of the integral, Warsaw, 1937, pp. 320-330.

2. - Sur le problème de mesure, Fund. Math. vol. 4 (1923) pp. 1-34.

3. - Théorie des operations lineaires, Warsaw, 1932.

4. G. Birkhoff, Integration of functions with values in a Banach space, Trans. Amer. Math. Soc. vol. 38 (1935) pp. 357-378.

5. - Lattice theory, Amer. Math. Soc. Colloquium Publications, vol. 25, rev. ed., 1948.

6. S. Bochner, Integration von Funktionen deren Werte die Elemente eines Vektorraumes sind, Fund. Math. vol. 20 (1935) pp. 262-276.

7. S. Bochner and K. Fan, Distributive order preserving operations in partially ordered vector sets, Ann. of Math. vol. 48 (1947) pp. 168-179.

8. S. Bochner and A. E. Taylor, Linear functionals on certain spaces of abstractly valued functions, Ann. of Math. vol. 39 (1938) pp. 913-944.

9. J. C. Burkill, Functions of intervals, Proc. London Math. Soc. (2) vol. 22 (1924) pp. 275-310.

10. C. Carathéodory, Entwurf fuer eine Algebraisierung des Integralbegriffs, S.-B. Math. Nat. Abt. Bayer. Akad. Wiss. (1938) pp. 27-68.

11. P. J. Daniell, A general form of integral, Ann. of Math. vol. 19 (1917-18) pp. 279-294.

12. - Further properties of the general integral, Ann. of Math. vol. 22 (1919-20) pp. 203-220.

13. J. Dieudonné, Sur le théorème de Lebesgue Nikodym, I. Ann. of Math. vol. 42 (1941) pp. 547-555; II. Bull. Soc. Math. France vol. 72 (1944) pp. 193-239; III. Ann. de l'Univ. de Grenoble vol. 23 (1948) pp. 25-53.

14. N. Dunford, Integration of abstract functions (Abstract), Bull. Amer. Math. Soc. vol. 41 (1936) p. 178.

15. Integration in general analysis, Trans. Amer. Math. Soc. vol. 37 (1935) pp. 441-453.

16. - Integration of vector valued functions (Abstract), Bull. Amer. Math. Soc. vol. 42 (1937) p. 24.

17. - Uniformity in linear spaces, Trans. Amer. Math. Soc. vol. 44 (1938) pp. $305-356$. 
18. G. Fichtenholz and L. Kantorovic, Sur les opérations dans l'espace des fonctions bornées, Studia Math. vol. 5 (1934) pp. 69-98.

19. M. Fréchet, L'intégrale abstrait d'une fonction abstraite d'une variable abstraite et son application d la moyenne d'un élément aléatoire de nature quelconque, Revue Sci. (Rev. Rose) vol. 82 (1944) pp. 483-512.

20. - Sur l'intégrale d'une fonctionnelle étendue à un ensemble abstrait, Bull. Soc. Math. France vol. 43 (1915) pp. 249-267.

21. H. Freudenthal, Teilweise geordnete Moduln, Neder. Akad. Wetensch. vol. 39 (1936) pp. 641-651.

22. I. Gelfand, Sur un lemme de la théorie des espaces linéaires, Comm. Inst. Sci. Math. de Kharkoff (4) vol. 13 (1936) pp. 35-40.

23. H. H. Goldstine, Linear functionals and integrals in abstract spaces, Bull. Amer. Math. Soc. vol. 47 (1941) pp. 615-621.

24. A. P. Gomes, Introduৎ̧̃oao estudo duma noção de funcional em espaços sem pontos, Portug. Mat. vol. 5 (1946) pp. 1-120. 206.

25. R. L. Gomes, Sur la notion de fonctionelle, Portug. Mat. vol. 5 (1946) pp. 202-

26. M. Gowurin, Ueber die Stieltjessche Integration abstrakter Funktionen, Fund. Math. vol. 27 (1936) pp. 255-268.

27. L. M. Graves, Riemann integration and Taylor's theorem in general analysis, Trans. Amer. Math. Soc. vol. 29 (1927) pp. 163-177.

28. H. Hahn, Ueber eine Verallgemeinerung der Riemannschen Integraldefinition, Monatsh. Math. Phys. vol. 26 (1915) pp. 3-18.

29. P. R. Halmos, Measure theory, New York, Van Nostrand, 1950.

30. T. H. Hildebrandt, Bounded linear functional operations, Trans. Amer. Math. Soc. vol. 36 (1934) pp. 868-875.

31. - Lebesgue integration in general analysis (Abstract), Bull. Amer. Math. Soc. vol. 33 (1927) p. 646.

32. S. Izumi, An abstract integral, I. Proc. Imp. Acad. Tokyo vol. 16 (1940) pp. 16-25; II. pp. 87-89; III. (w. M. Nakamura) pp. 518-523; IV. vol. 17 (1940) pp. 1-4; V. (w. N. Matuyama and M. Orihara) vol. 18 (1942) pp. 45-49; VII. vol. 18 (1942) pp. 53-56; X. pp. 543-547.

33. R. L. Jeffery, Integration in abstract space, Duke Math. J. vol. 6 (1940) pp. 706-718.

34. L. Kantorovic, Linear halbgeordnete Raeume, Mat. Sbornik. (2) vol. 44 (1937) pp. 121-165.

35. D. A. Kappos, Ein Beitrag zur Carathéodoryschen Definition der Ortsfunktionen in Booleschen Algebren, Math. Zeit. vol. 51 (1948) pp. 616-634.

36. A. Kolmogoroff, Untersuchungen ueber den Integralbegriff, Math. Ann. vol. 103 (1930) pp. 654-696.

37. - Z Zur Normierbarkeit eines allgemeinen topologischen linearen Raumes, Studia Math. vol. 5 (1934) pp. 29-33.

38. K. Kunisawa, Integrations in a Banach space, Proc. Phys. Math. Soc. Japan (3) vol. 25 (1943) pp. 524-529.

39. H. Lebesgue, Leçons sur l'intégration, 2d ed., Paris, 1928.

40. H. M. MacNeille, Extensions of measure, Proc. Nat. Acad. Sci. U.S.A. vol. 24 (1938) pp. 188-193.

41. - Unified theory of integration, Proc. Nat. Acad. Sci. U.S.A. vol. 27 (1941) pp. 71-76.

42. M. S. MacPhail, Integration of functions in a Banach space, Nat. Math. Mag. vol. 20 (1945) pp. 69-78. 
43. E. J. McShane, Remark concerning integration, Proc. Nat. Acad. Sci. U.S.A. vol. 35 (1949) pp. 46-49.

44. N. Matsuyama, An abstract integral IX, Proc. Imp. Acad. Tokyo vol. 18 (1942) pp. 539-542.

45. E. H. Moore and H. L. Smith, $A$ general theory of limits, Amer. J. Math. vol. 44 (1922) pp. 102-121.

46. M. Nakamura, An abstract integral VI, Proc. Imp. Acad. Tokyo vol. 18 (1942) pp. 50-52.

47. $\mathrm{H}$. Nakano, Ueber Erweiterungen von allgemein teilweise geordneten Moduln, I. Proc. Imp. Acad. Tokyo vol. 18 (1942) pp. 626-630; II. ibid. vol. 19 (1943) pp. 138143.

48. J. v. Neumann, On complete topological spaces, Trans. Amer. Math. Soc. vol. 37 (1935) pp. 1-20.

49. O. Nikodym, Sur une génêralisation des intégrales de M. J. Radon, Fund. Math. vol. 15 (1930) pp. 131-179.

50. J. H. M. Olmsted, Lebesgue theory on a Boolean algebra, Trans. Amer. Math. Soc. vol. 51 (1942) pp. 164-193.

51. M. Orihara and G. Sunouchi, An abstract integral VIII, Proc. Imp. Acad. Tokyo vol. 18 (1942) pp. 535-538.

52. B. J. Pettis, On integration in vector spaces, Trans. Amer. Math. Soc. vol. 44 (1938) pp. 277-304.

53. R. S. Phillips, Integration in a convex linear topological space, Trans. Amer. Math. Soc. vol. 47 (1940) pp. 114-145.

54. G. B. Price, The theory of integration, Trans. Amer. Math. Soc. vol. 47 (1940) pp. 1-51.

55. C. E. Rickart, An abstract Radon-Nikodym theorem, Trans. Amer. Math. Soc. vol. 56 (1944) pp. 50-66.

56. - Integration in a convex linear topological space, Trans. Amer. Math. Soc. vol. 52 (1942) pp. 498-521.

57. J. Ridder, Mass- und Integrationstheorie in Strukturen, I, Acta. Math. vol. 73 (1941) pp. 131-173; II. Neder. Akad. Wetensch. vol. 49 (1946) pp. 167-174.

58. F. Riesz, Sur l'intégrale de Lebesgue, Acta Math. vol. 42 (1919) pp. 191-205.

59. - Sur quelques notions fondamentales dans la théorie des operations linéaires, Ann. of Math. vol. 41 (1940) pp. 174-206.

60. S. Saks, Integration in abstract metric spaces, Duke Math. J. vol. 4 (1938) pp. 408-411.

61. M. H. Stone, Notes on integration, Proc. Nat. Acad. Sci. U.S.A., I. vol. 34 (1948) pp. 336-342; II. pp. 447-455; III. pp. 483-490; IV. vol. 35 (1949) pp. 50-58.

62. G. Vanderlijn, Une généralisation de l'intégrale de Radon, Bull. Soc. Roy. Sci. Liège vol. 10 (1941) pp. 168-175.

63. B. Vulikh, Sur l'intégrale de Stieltjes des fonctions, dont les valeurs appartiennent $d$ un espace semiordonné, Leningrad. State Univ. Ann. [Math. ser. 12] vol. 83 (1941) pp. 3-29.

64. F. Wecken, Abstrakte Integrale und fastperiodische Funktionen, Math. Zeit. vol. 45 (1939) pp. 377-404.

65. J. V. Wehausen, Transformations in linear topological spaces, Duke Math. J. vol. 4 (1938) pp. 157-169.

\section{UNIVERSITY OF MichigaN}

